\title{
CONCERNING THE FUNCTION EQUATION $f(g)=f$, REGULAR MAPPINGS AND PERIODIC MAPPINGS
}

\author{
SAM W. YOUNG
}

\begin{abstract}
Under certain conditions on the space $X$, given $f: X \rightarrow Y$ is light and $g: X \rightarrow X$, the equation $f(g)=f$ yields only periodic solutions for $g$.
\end{abstract}

1. Introduction. In $[\mathbf{M}$, p. 238], Mioduszewski proved the following theorem: If each of $f$ and $g$ is a mapping of $[0,1]$ onto itself, $f$ is light and $f g=f$, then $g^{2}=$ id. Thus $g$ is either the identity or an involution, the only types of periodic maps on $[0,1]$. In this paper we explore more general conditions under which the equation $f g=f$ leads to the conclusion that $g$ is periodic. These results are contained in Theorem 3.1. In $\S 2$ we establish some preliminary theorems. $\S 4$ contains related examples.

All spaces considered are compact metric and $\rho$ denotes the sup metric on function spaces. A mapping $f: X \rightarrow Y$ is called light iff each point inverse is totally disconnected. A mapping $f: X \rightarrow X$ is called positively regular if the family of iterates $\left\{f, f^{2}, f^{3}, \ldots\right\}$ is an equicontinuous family. If $f$ is a homeomorphism, then $f$ is regular iff the family of all iterates is equicontinuous. Also in case $f$ is a homeomorphism, positively regular implies regular because of compactness. Lemma 2.2 will strengthen this. A mapping $f: X \rightarrow X$ is periodic iff there exists a positive integer $n$ such that $f^{n}=$ id $=$ the identity mapping on $X$. The smallest such $n$ is the period of $f$. The double arrow denotes an onto mapping.

2. Preliminaries. In this section we establish the connection between the function equation $f g=f$ and regular homeomorphisms.

LEMMA 2.1. If $g$ is a positively regular mapping of a compact metric space $X$ onto itself, then either $g$ is a homeomorphism or there exists $\delta>0$ such that $\rho\left(g^{i}\right.$, id $) \geq \delta$ for $i=1,2,3, \ldots$.

PROOF. If $g$ is not a homeomorphism then there exists $c \in X$ such that $g^{-1}(c)$ is nondegenerate. Since the family $\left\{g, g^{2}, g^{3}, \ldots\right\}$ is equicontinuous, no subsequence of $\operatorname{diam}\left(g^{-1}(c)\right), \operatorname{diam}\left(g^{-2}(c)\right), \operatorname{diam}\left(g^{-3}(c)\right), \ldots$ converges to 0 . It follows that there exists $\delta>0$ and points $a_{i}, b_{i} \in X$ such that $g^{i}\left(a_{i}\right)=g^{i}\left(b_{i}\right)=c$ and $d\left(a_{i}, b_{i}\right) \geq 2 \delta$, $i=1,2,3, \ldots$. Then for each $i$,

$$
\begin{aligned}
\rho\left(g^{i}, \mathrm{id}\right) & \geq \operatorname{Max}\left\{d\left(g^{i}\left(a_{i}\right), \operatorname{id}\left(a_{i}\right)\right), d\left(g^{i}\left(b_{i}\right), \operatorname{id}\left(b_{i}\right)\right)\right\} \\
& =\operatorname{Max}\left\{d\left(c, a_{i}\right), d\left(c, b_{i}\right)\right\} \geq \delta .
\end{aligned}
$$

It should be pointed out here that on compact metric spaces, a homeomorphism is regular iff it is almost periodic [GH].

Received by the editors April 26, 1985 and, in revised form, June 20, 1985.

1980 Mathematics Subject Classification. Primary 54C10, 54F62; Secondary 54F20, 57S10.

Key words and phrases. Function equation, regular mapping, periodic mapping, light mapping, finite graph, orientable surface. 
LEMMA 2.2. If $g$ is a positively regular mapping of a compact metric space $X$ onto itself, then $g$ is a regular homeomorphism.

PROOF. We can regard the space of all mappings of $X$ onto $X$ as a topological semigroup using the composition operation and the metric $\rho$. By the Ascoli theorem, $\Gamma(g)=\operatorname{cl}\left\{g, g^{2}, g^{3}, \ldots\right\}$ is a compact subset and so in fact is a compact subsemigroup. By a theorem of Numakura [N, Lemma 3, p. 102], $\Gamma(g) \supset K(g)=$ the set of all cluster points of the sequence $\left\{g, g^{2}, g^{3}, \ldots\right\}$ is a (an abelian) group. The unit $e$ of $K(g)$ is an idempotent, $e^{2}=e$. Since $e$ is a mapping of $X$ onto $X$, it follows that $e=\mathrm{id}$. And since id is the limit of a subsequence of $\left\{g, g^{2}, g^{3}, \ldots\right\}$ it follows from Lemma 2.1 that $g$ is a homeomorphism.

LEMMA 2.3. If each of $X$ and $Y$ is a locally connected compact metric space and $f: X \rightarrow Y$ is a light mapping, then the family of mappings $\{g: g: X \rightarrow X$ and $f g=f\}$ is equicontinuous.

Proof. Suppose to the contrary. Then there exists $\varepsilon_{0}>0, a_{i}, b_{i} \in X$ and $g_{i}$ such that $f g_{i}=f, d\left(a_{i}, b_{i}\right)<1 / i$ and $d\left(g_{i}\left(a_{i}\right), g_{i}\left(b_{i}\right)\right) \geq \varepsilon_{0}, i=1,2,3, \ldots$ Wlog assume that there exists $P \in X$ such that $a_{i} \rightarrow P$ and $b_{i} \rightarrow P$. Since $X$ is locally connected at $P$, it follows that there exists a sequence of continua $A_{1} \supset A_{2} \supset A_{3} \supset$ .. $\supset\{P\}$ closing down on $P$ such that, for each $i, \operatorname{diam}\left(g_{i}\left(A_{i}\right)\right) \geq \varepsilon_{0}$. Again wlog assume that there is a nondegenerate continuum $K \subset X$ such that $g_{i}\left(A_{i}\right) \rightarrow K$.

Now we have $f g_{i}\left(A_{i}\right) \rightarrow f(K)$ but since $f g_{i}\left(A_{i}\right)=f\left(A_{i}\right) \rightarrow f(P)$ it follows that $f(K)=f(P)$. This is not possible since $f$ is light.

THEOREM 2.4. If each $X$ and $Y$ is a locally connected compact metric space, $f: X \rightarrow Y$ is a light mapping and $g: X \rightarrow X$ is a mapping such that $f g=f$, then $g$ is a regular homeomorphism such that for each $x \in X$ the closure of the $g$-orbit of $x$ is totally disconnected.

ProOF. Suppose that $X, Y, f$ and $g$ are given and satisfy the hypothesis. Since for each $i=1,2,3, \ldots, f g^{i}=f$, it follows from Lemma 2.3 and Lemma 2.2 that $g$ is a regular homeomorphism.

Suppose $x \in X$ and consider $O(x)=\left\{g^{i}(x): i=0, \pm 1, \pm 2, \ldots\right\}$, the $g$-orbit of $x$. We have that, for each integer $i, f g^{i}(x)=f(x)$ and so $f(\operatorname{cl}(O(x)))=f(x)$. Since $f$ is light, $\operatorname{cl}(O(x)) \subset f^{-1} f(x)$ and must be totally disconnected.

3. The main theorem. The compact metric space $G$ is a finite graph iff $V=\{x: x \in G$ and order $(x) \neq 2\}$ is finite, $G \sim V$ has finitely many components and if $C$ is a component of $G \sim V$ then $\operatorname{cl}(C)$ is either an arc or a simple closed curve.

Part (1) of Theorem 3.1 establishes a direct generalization of the theorem of Mioduszewski $[\mathbf{M}]$ referred to in the introduction. Parts (2) and (3) give similar results for some 2-dimensional spaces.

THEOREM 3.1. Suppose each of $X$ and $Y$ is a metric space, $f: X \rightarrow Y$ is a light mapping and $g: X \rightarrow X$ is a mapping such that $f g=f$. Then $g$ is a periodic homeomorphism if $X$ satisfies one of the following:

(1) $X$ is a finite graph.

(2) $X$ is a compact subset of the plane whose boundary is a finite graph.

(3) $X$ is a compact connected orientable surface. 
PROOF OF PART (1). By Theorem 2.4, $g$ is a regular homeomorphism such that the closure of the orbit of each point is totally disconnected. We will first consider the special case that $X$ is a simple closed curve. There are three possibilities $[\mathbf{H}$, p. 129]: (a) If $g$ is order preserving and $X$ has a fixed point under $g$, then $g$ is the identity on $X$. (b) If $g$ is order preserving and $X$ has no periodic point under $g$, then $g$ is topologically an irrational rotation on $X$. (c) If $g$ is not order preserving, then $g^{2}=$ id. Case (b) is eliminated since the orbit of any point in $X$ would be dense in $X$, and case (c) gives $g^{2}=\mathrm{id}$. The remaining case is (a) with some point of period $n$ and here we have $g^{n}=\mathrm{id}$.

Now for the general case let

$$
V=\{x: x \in X \text { and order }(x) \neq 2\}
$$

and

$$
\mathrm{C}=\{A: A \text { is a component of } X \sim V\} \text {. }
$$

Both $V$ and $C$ are finite and the homeomorphism $g$ permutes the elements of $V$ and the elements of $C$. There exists a positive integer $m$ such that $g^{m}$ takes each point of $V$ onto itself and each member of $C$ onto itself. If $A \in C$, there exists a positive integer $n(A)$ such that $g^{n(A)}=$ id on $\operatorname{cl}(A)$. This is because the theorem of Mioduszewski $[\mathbf{M}]$ handles the case that $\operatorname{cl}(A)$ is an arc and the argument given above handles the case that $\operatorname{cl}(A)$ is a simple closed curve. Let $n$ be a common multiple of $m$ and $\{n(A): A \in C\}$ and then $g^{n}=$ id on $X$.

PROOF OF PART (2). Let us suppose that int $(X) \neq \varnothing$ since otherwise part (1) would apply. Under the hypothesis, $X$ would be locally connected and so by Theorem $2.4 \mathrm{~g}$ is a regular homeomorphism such that the closure of the orbit of each point is totally disconnected. In $[\mathbf{H}$, p. 127] it is shown that each component of $\operatorname{int}(X)$ contains a simple closed curve which is invariant under some positive interate of $g$. Let $K$ be a simple closed curve lying in a component $A$ of $\operatorname{int}(X)$ and $m$ be a positive integer such that $g^{m}(K)=K$. With the mappings $f$ and $g^{m}$ restricted to $K, f g^{m}=f$ and so, by part (1), $g^{m}$ is periodic on $K$.

It is also shown in $[\mathbf{H}$, p. 129] that if $K$ is a simple closed curve contained in $\operatorname{int}(X)$ and if both $K$ and some point of $K$ are invariant under a positive iterate of $g$, then $g$ is periodic on the component of $X$ which contains $K$. Applying this theorem to $g^{m}$, we have that $g^{m}$ is periodic on $A$ and therefore $g$ is periodic on $A$. For each component $A$ of $\operatorname{int}(X)$ let $n(A)$ be a positive integer such that $g$ restricted to $A$ is of period $n(A)$. Clearly also, $g$ is of period $n(A)$ on $\operatorname{cl}(A)$.

Now bd $(X)$ is invariant under $g$ and, with the mappings $f$ and $g$ restricted to bd $(X), f g=f$. By part (1) there is a positive integer $l$ such that $g$ is of period $l$ restricted to $\mathrm{bd}(X)$. Let $n$ be a common multiple of $l$ and $\{n(A): A$ is a component of $\operatorname{int}(X)\}$. Then $g^{n}=\operatorname{id}$ on $X$.

PROOF OF PART (3). By Theorem 2.4, $g$ is a regular homeomorphism such that the closure of the orbit of each point is totally disconnected. The theorem of von Kerekjarto [K1] says that $g$ is periodic if $X$ is anything except a 2-sphere, torus, disc or annulus. If $X$ is a disc or annulus, then part (2) applies (or for a separate argument see [F and $\mathbf{R}]$ ).

The regular homeomorphisms on the torus are characterized $[\mathbf{K 2}]$ as being topologically equivalent to a product of standard rotations and such a homeomorphism 
would either be periodic, or rotate an invariant simple closed curve $K$ with a dense orbit in $K$ or have a dense orbit. The latter cases cannot occur by Theorem 2.4.

The regular homeomorphisms on the 2-sphere are characterized $[\mathbf{R}]$ as being topologically equivalent to either a standard rotation or a reflection through the equator or a reflection through the equator followed by a standard rotation. The second type is periodic and each of the other types rotates an invariant simple closed curve $[\mathbf{R}]$. In this case, as in the case of the torus, the rotation on the invariant simple closed curve must be a periodic rotation with some period $n$. The period of $g$ would be $n$ in all cases except for a reflection followed by a rotation on the 2 -sphere. In this case the period of $g$ would be $n$ if $n$ is even and $2 n$ if $n$ is odd. This completes the proof.

The possibility of extending Theorem 3.1 to include cases of dimension greater than 2 seems difficult since very little is known about regular homeomorphisms in this setting. One approach to the study of regular homeomorphisms in dimension 3 can be found in $[\mathbf{B}]$.

4. Some examples. If $X$ is a compact metric space and $g: X \rightarrow X$ is periodic, then let $Y$ be the space obtained by decomposing $X$ into $g$-orbits. If $f: X \rightarrow Y$ is the projection map then of course $f g=f$ and, for any $h: Y \rightarrow Y, h f g=h f$. In a similar manner we construct the following

EXAMPLE 4.1. Let $X$ be the locally connected space which is the union of a countably collection of arcs $\left[a, b_{1}\right],\left[a, b_{2}\right],\left[a, b_{3}\right], \ldots$ which have only the point $a$ in common and such that $\operatorname{diam}\left(\left[a, b_{1}\right]\right) \rightarrow 0$ as $i \rightarrow \infty$. Let $g: X \rightarrow X$ be the mapping such that $g$ fixes $a$ and permutes the first two arcs $\left[a, b_{1}\right]$ and $\left[a, b_{2}\right]$, permutes the next three arcs with period three, the next four with period four and so on.

Let $Y$ be the space obtained by decomposing $X$ into $g$-orbits. We see that $Y$ is homeomorphic to $X$. If $f: X \rightarrow Y$ is the projection map, then $f g=f$. The mapping $g$ is regular and pointwise periodic but not periodic. This example indicates that some sort of finite structure is necessary on $X$ to conclude that $g$ is periodic.

EXAMPLE 4.2. We use $X, Y, f$ and $g$ from Example 4.1. Note that the Hilbert Cube $Q \simeq X \times X \times X \times \cdots$. Let $g^{\infty}: Q \rightarrow Q$ be the product $g \times g \times g \times \cdots$ and let $f^{\infty}: Q \rightarrow Q$ be $f \times f \times f \times \cdots$. We have that $f g=f$ and now $g$ is regular but not pointwise periodic.

EXAMPLE 4.3. Let $f:[0,1] \rightarrow[0,1]$ be any mapping which is not light. Let us say that $[a, b]$ is a proper subinterval of $[0,1]$ such that $f([a, b])=c \in[0,1]$. Then let $g:[0,1] \rightarrow[0,1]$ be any mapping such that $g:[a, b] \rightarrow[a, b]$ and $g(x)=x$ for all $x \notin[a, b]$. Then $f g=f$. This example indicates that the requirement that $f$ be light is necessary to conclude that $g$ is periodic.

\section{REFERENCES}

[B] B. L. Brechner, Prime ends and group actions, Topology Proc. (to appear).

[F] N. E. Foland, A characterization of the almost periodic homeomorphisms on the closed 2-cell, Proc. Amer. Math. Soc. 16 (1965), 1031-1034.

[GH] W. H. Gottschalk and G. A. Hedlund, Topological dynamics, Amer. Math. Soc. Colloq Publ., Vol. 36, Amer. Math. Soc., Providence R. I., 1956.

[H] E. Hemmingsen, Plane continua admitting non-periodic autohomeomorphisms with equicontinuous interates, Math. Scand. 2 (1954), 119-141. 
[K1] B. von Kerekjarto, Über regulare Abbildingen von Flachen auf sich, Acta. Sci. Math. (Szeged) 7 (1934), 65-75.

[K2] _ , Über die regularen Abbildungen des Torus, Acta. Sci. Math. (Szeged) 7 (1934), 76-84.

[M] J. Mioduszewski, On a quasi-ordering in the class of continuous mappings of a closed interval into itself, Colloq. Math. 9 (1962), 232-240.

[N] K. Numakura, On bicompact semigroups, Math. J. Okayama Univ. 1 (1952), 99-108.

[R] G. K. Ritter, A characterization of almost periodic homeomorphisms on the 2-sphere and the annulus, Topology Appl. 9 (1978), 185-191.

Department of Mathematics, Auburn University, Auburn, Alabama 36849 